\title{
A New Model Incorporating Axillary Ultrasound After Neoadjuvant Chemotherapy to Predict Non-Sentinel Lymph Node Metastasis in Invasive Breast Cancer
}

\section{Kai Zhang* \\ Qian Zhu* \\ Danli Sheng \\ Jiawei Li \\ Cai Chang}

Department of Medical Ultrasound, Fudan University Shanghai Cancer

Center, Shanghai, People's Republic of China

*These authors contributed equally to this work
This article was published in the following Dove Press journal:

Cancer Management and Research

Purpose: Few models with good discriminative power have been introduced to predict the risk of non-sentinel lymph node (non-SLN) metastasis in breast cancer after neoadjuvant chemotherapy (NAC). We aimed to develop a new and simple model for predicting the probability of non-SLN metastasis in breast cancer and facilitate the selection of patients who could avoid unnecessary axillary lymph node dissection following NAC.

Patients and Methods: A total of 298 patients diagnosed with invasive breast cancer, who underwent SLN biopsy after completing NAC and subsequently breast surgery, were included and classified into the training set $(n=228)$ and testing set $(n=70)$. Univariate and multivariate analyses were used to select factors that could be determined prior to breast surgery and significantly correlated with non-SLN metastasis in the training set. A logistic regression model was developed based on these factors and validated in the testing set.

Results: Nodal status before NAC, post-NAC axillary ultrasound status, SLN number, and SLN metastasis number were independent predictors of non-SLN metastases in breast cancer after NAC. A predictive model based on these factors yielded an area under the curve of 0.838 (95\% confidence interval: $0.774-0.902, p<0.001$ ) in the training set. When applied to the testing set, this model yielded an area under the curve of 0.808 (95\% confidence interval: $0.609-1.000, p=0.003)$.

Conclusion: A new and simple model, which incorporated factors that could be determined prior to breast surgery, was developed to predict non-SLN metastasis in invasive breast cancer following NAC. Although this model performed excellently in internal validation, it requires external validation before it can be widely utilized in the clinical setting.

Keywords: breast carcinoma, neoadjuvant chemotherapy, sentinel lymph node, ultrasonography

\section{Introduction}

Axillary lymph node tumor burden has a critical impact on the treatment decision and prognosis in patients with breast cancer. ${ }^{1}$ Therefore, it is important to determine the status of axillary lymph nodes prior to surgery. Usually, patients with clinical positive $(\mathrm{cN}+)$ axillary lymph node directly undergo axillary lymph node dissection (ALND). Sentinel lymph node biopsy (SLNB) is the method used to identify axillary lymph node metastasis in patients with $\mathrm{cN} 0$ breast cancer who have negative results in axillary imaging or suspected metastasis in axillary imaging, but ultrasound-guided (US-guided) fine needle aspiration (FNA) did not detect
Correspondence: Cai Chang

Fudan University Shanghai Cancer Center, No. 270, Dong'an Road, Xuhui District, Shanghai 200032, People's Republic of China

Tel $+86180 \quad 173 \mid 2605$

Fax +862164174774

Email changc62@I26.com 
cancer cells. ${ }^{2}$ Positive SLNB results are commonly followed by ALND, which may cause more serious and a greater number of side effects than SLNB alone. ${ }^{3}$ Patients with negative SLNB results can safely avoid ALND, since the false negative rate (FNR) of SLNB, meaning that SLNB is negative but follow-up ALND finds metastasis in axillary non-sentinel lymph nodes (nonSLN), is no more than $10 \%,{ }^{4}$ which is the clinically acceptable threshold.

Neoadjuvant chemotherapy (NAC) has been increasingly used in patients with operable breast cancer. Following the publication of several prospective multicentric clinical trials evaluating the effectiveness of SLNB in patients with breast cancer who received NAC, ${ }^{5-7}$ SLNB has been increasingly performed in the setting of NAC. ${ }^{8,9}$ However, the reported FNRs varied $0-20 \%$ in patients with initial $\mathrm{cN} 0$, and $7-30 \%$ in patients with initial $\mathrm{cN}+{ }^{10-15}$ Furthermore, according to previous reports, metastasis in non-SLNs is not detected in $46-68 \%$ of patients with positive SLNs. ${ }^{5-7}$ The above data suggest that: 1) SLNB alone may not reliably discriminate patients who could safely avoid further ALND in the setting of NAC; and 2) more than half of the patients have to undergo unnecessary ALND, if the decision is based only on positive SLNB. Therefore, several models have been developed to predict the risk of non-SLN metastasis in patients with breast cancer receiving NAC. ${ }^{16-18}$ Unfortunately, these models have limited value with an adjusted area under the receiver operating characteristic curve (AUC) $<0.8$. Thus, the development of a new predictive model with excellent discriminatory power is warranted.

Axillary ultrasound (AUS) is a convenient and useful imaging method for clinical axilla staging prior to surgery in breast cancer patients with or without NAC. ${ }^{19-21}$ Thus, numerous institutions have adopted AUS as a routine practice to evaluate axillary status prior to surgery in the management of patients with breast cancer. However, to the best of our knowledge, there has been no model including pre-surgery AUS to predict the risk of nonSLN metastasis in patients after NAC. Therefore, this retrospective study aimed to develop such a model in this setting.

\section{Patients and Methods}

\section{Patients}

We performed a retrospective review using the digital medical records of patients from January 2013 to January 2018. Patients who met the following criteria were included in the analysis: 1) initial pathological diagnosis of invasive breast cancer; 2) NAC and subsequent mastectomy or breastconserving surgery; 3) US assessment of the axilla after completing NAC; and 4) SLNB after completing NAC and prior to breast surgery. Patients with distant metastasis and breast cancer combined with other malignancies were excluded. Finally, 298 eligible patients were included in this study. We allocated 228 and 70 patients to the training and testing sets, respectively. The training set was used to evaluate factors affecting the non-SLN status and develop a predictive model. This predictive model was subsequently validated using the testing set.

The information obtained from the physical examination, magnetic resonance imaging, US, and mammography prior to and after NAC was used to determine the tumor size and axilla lymph node metastasis. The clinical $\mathrm{T}$ stage was classified according to the 8th American Joint Committee on Cancer, while the clinical tumor response to NAC was classified according to the Response Evaluation Criteria In Solid Tumors guideline (version 1.1). Complete response was defined as complete remission of all known diseases. Partial response was defined as $30 \%$ reduction in the maximal diameter of the breast tumor without progression of any lesion or appearance of new disease. Progressive disease was defined as a $20 \%$ increase in the maximal diameter and/or the appearance of new lesions. Tumors with change between partial response and progressive disease were classified as stable disease. All included patients underwent FNA before NAC to confirm axilla lymph node metastasis. The expression status of estrogen receptor (ER), progesterone receptor (PR), Ki67 , and human epidermal growth factor receptor 2 (HER2) was determined through immunohistochemical staining. Hormone receptor (HR) positivity was defined as $>1 \%$ of cells staining for ER and/or PR. Otherwise, tumors were defined as HR negative. For Ki-67, $<14 \%$ and $>14 \%$ of tumor cells staining positive denoted low and high expression, respectively. Tumors with HER2 scores of $3+$ were considered positive, whereas those with scores of $1+$ or 0 were considered negative. In tumors with $2+$ scores, fluorescence in situ hybridization was used to determine HER2 amplification. The tumors were classified into four subtypes as follows: luminal A (HR positive, HER2 negative, Ki-67 low expression), luminal B (HR positive, HER2 negative, Ki-67 high expression or HR positive, HER2 positive), HER2 enriched (HR negative, HER2 positive), and basal-like (HR negative, HER2 negative). 


\section{US Assessment of Axilla}

The AUS was routinely performed after whole-breast US by a single physician using the Logic E9 (GE Healthcare, Kretz, Zipf, Austria), IU22 (Philips Medical Systems, Bothell, WA, USA), Aixplorer (Supersonic Imaging, Aixen-Provence, France), Aplio 500 (Toshiba medical system, Japan), or Mylab90 (Esaote, Genoa, Italy) equipped with a $5-14-\mathrm{MHz}$ linear-array transducer.

The results of the AUS were categorized as either positive or negative. AUS was considered positive when an abnormal node with at least one of the following suspicious findings was recorded: diffuse cortical thickening of $\geq 3 \mathrm{~mm}$; focal cortical bulge; eccentric cortical thickening; rounded hypoechoic node; complete or partial effacement of the fatty hilum; nonhilar cortical blood flow on color Doppler images; complete or partial replacement of the node with an ill-defined or irregular mass; or microcalcifications in the node. Otherwise, the AUS was considered negative.

\section{SLNB Method}

SLN mapping was conducted using vital blue (1\% indigo carmine). Following the injection of $5 \mathrm{~mL} 1 \%$ indigo carmine, the breast was massaged for $5 \mathrm{~min}$. All blue and palpable lymph nodes were excised and submitted as SLNs. The SLNs were initially evaluated through touch imprint cytology. Subsequently, the SLNs were fixed in $10 \%$ formalin, embedded in paraffin, and subjected to hematoxylin and eosin staining. The largest metastases in the SLN with a maximal diameter $>2 \mathrm{~mm}, 0.2-2 \mathrm{~mm}$, and $\leq 0.2 \mathrm{~mm}$ were classified as macrometastases, micrometastases, and isolated tumor cell, respectively. Patients with metastatic SLNs detected using touch imprints routinely underwent immediate ALND during the initial surgery period. Occasionally, patients with tumor-free touch imprints but node-positive permanent sections underwent subsequent ALND, unless they refused to undergo a second surgery.

\section{Evaluation of Non-SLN Metastasis}

Nodes removed from ALND were submitted as non-SLNs and subjected to histopathologic examination. If any lymph node metastases were detected during ALND, a non-SLN metastasis was considered to exist. Otherwise, the patient was considered free of non-SLN metastasis. For patients who did not undergo ALND, the metastatic status of non-SLNs was defined by means of follow-up imaging information. If lymph nodes were judged as positive at follow-up imaging surveillance with
AUS within 6 months and US-guided FNAB reported that metastasis from breast cancer or atypical cells, a non-SLN metastasis was considered to exist. Otherwise, the patient was considered free of non-SLN metastasis.

\section{Statistical Analysis}

Quantitative data were expressed as median and range, while qualitative data were presented as frequency and percentage. Univariate analysis was used to identify the risk factors of non-SLN metastasis. Student's $t$ test or Mann-Whitney $U$-test was used for the analysis of quantitative data, while the Chi-squared test was employed to analyze qualitative data. All variables with $p<0.05$ in the univariate analysis were subsequently included in a multivariate analysis. A multivariate logistic regression backward stepwise method was used to develop a model for the prediction of non-SLN metastasis in patients after NAC. Variables with $p<0.05$ in the multiple analysis were included in the final predictive model. The AUC was calculated to quantify the ability of the model to rank patients according to risk. All tests were two-sided, and $p<0.05$ indicated statistical significance. The SPSS version 19.0 (IBM Corp., Armonk, NY, USA) software was used for statistical analysis.

\section{Results}

\section{Patients' Characteristics}

The baseline characteristics of patients included in the training set are shown in Table 1. Most patients (219/ 228) received NAC with an anthracycline- and taxanebased regimen; trastuzumab was added to the regimen for 116 patients with HER2-positive status. Of the 228 patients, 95 patients $(41.7 \%)$ had breast-conserving surgery after completing NAC, whereas the remaining patients underwent mastectomy. Prior to NAC, 133 patients $(58.3 \%, 133 / 228)$ were confirmed as $\mathrm{cN}+$. Post NAC, AUS detected suspicious lymph node metastasis in 21 patients, among whom 17 were classified as $\mathrm{cN}+$ prior to NAC. The SLNB procedure was successfully performed in all patients with at least one lymph node identified. Residual disease was identified in the SLNs (SLN+) of 65 patients $(28.5 \%, 65 / 228)$, including 11 and one patients with only micrometastasis and only isolated tumor cell, respectively. ALND was performed in 131 patients (57.5\%, 131/228), including $65 \mathrm{SLN}+$ and $66 \mathrm{SLN}-$ patients. Non-SLN metastasis was found in 48 patients; 13 of these metastases were confined to the non-SLNs. 
Table I Baseline Characteristics of Patients Included in the Training Set

\begin{tabular}{|c|c|c|c|c|}
\hline \multirow[t]{2}{*}{ Characteristics } & \multirow{2}{*}{$\begin{array}{l}\text { Total } \\
\text { Number } \\
(\%)\end{array}$} & \multicolumn{2}{|c|}{$\begin{array}{l}\text { Non-SLN Metastasis } \\
\text { (\%) }\end{array}$} & \multirow[t]{2}{*}{$p$} \\
\hline & & No & Yes & \\
\hline Total & $228(100)$ & $180(78.9)$ & $48(21.1)$ & \\
\hline Age, median (range) & $48(25-77)$ & $48(25-76)$ & $47(27-77)$ & 0.723 \\
\hline Menopause & & & & 0.402 \\
\hline No & $112(49.1)$ & 91 (50.6) & $21(43.8)$ & \\
\hline Yes & $116(50.9)$ & $89(49.4)$ & $27(56.2)$ & \\
\hline Pathological types & & & & 0.311 \\
\hline IDC & $214(93.9)$ & $167(92.8)$ & $47(97.9)$ & \\
\hline Else & $14(6.1)$ & $13(7.2)$ & I (2.1) & \\
\hline cT & & & & 0.130 \\
\hline 1 & $44(19.3)$ & $36(20.0)$ & $8(16.7)$ & \\
\hline 2 & $163(71.5)$ & $13 \mid(72.8)$ & $32(66.6)$ & \\
\hline 3 & $21(9.2)$ & $13(7.2)$ & $8(16.7)$ & \\
\hline Pre-NAC cN & & & & 0.001 \\
\hline Negative & $95(41.7)$ & $85(47.2)$ & $10(20.8)$ & \\
\hline Positive & $133(58.3)$ & $95(52.8)$ & $38(79.2)$ & \\
\hline ER & & & & 0.001 \\
\hline Negative & $114(50.0)$ & $100(55.6)$ & $14(29.2)$ & \\
\hline Positive & $114(50.0)$ & $80(44.4)$ & $34(70.8)$ & \\
\hline PR & & & & 0.021 \\
\hline Negative & $142(62.3)$ & $119(66.1)$ & $23(47.9)$ & \\
\hline Positive & $86(37.7)$ & $61(33.9)$ & $25(52.1)$ & \\
\hline HER2 & & & & 0.644 \\
\hline Negative & $112(49.1)$ & $87(48.3)$ & $25(52.1)$ & \\
\hline Positive & $116(50.9)$ & $93(5 \mid .7)$ & $23(47.9)$ & \\
\hline $\mathrm{Ki}-67$ & & & & 0.044 \\
\hline Low & $21(9.2)$ & $13(7.2)$ & $8(16.7)$ & \\
\hline High & $207(90.8)$ & $167(92.8)$ & $40(83.3)$ & \\
\hline HR & & & & 0.008 \\
\hline Negative & $108(47.4)$ & $94(52.2)$ & $14(29.2)$ & \\
\hline Positive & $120(52.6)$ & $86(47.8)$ & $34(70.8)$ & \\
\hline Molecular type & & & & 0.019 \\
\hline Luminal A & $16(7.0)$ & $10(5.6)$ & $6(12.5)$ & \\
\hline Luminal B & $104(45.6)$ & $76(42.2)$ & $28(58.4)$ & \\
\hline HER2+ & $61(26.8)$ & $51(28.3)$ & $10(20.8)$ & \\
\hline Basal-like & $47(20.6)$ & $43(23.9)$ & $4(8.3)$ & \\
\hline Post-NAC AUS & & & & $<0.001$ \\
\hline Negative & $207(90.8)$ & $173(96.1)$ & $34(70.8)$ & \\
\hline Positive & $21(9.2)$ & $7(3.9)$ & $14(29.2)$ & \\
\hline cTumor response & & & & 0.806 \\
\hline $\mathrm{CR}$ & $44(19.3)$ & $35(19.4)$ & $9(18.8)$ & \\
\hline PR & $139(61.0)$ & $108(60.0)$ & $31(64.6)$ & \\
\hline SD and PD & 45 (19.7) & $37(20.6)$ & $8(16.7)$ & \\
\hline $\begin{array}{l}\text { SLN number, median } \\
\text { (range) }\end{array}$ & $3(1-8)$ & $3(I-8)$ & $3(1-7)$ & $<0.001$ \\
\hline
\end{tabular}

(Continued)
Table I (Continued).

\begin{tabular}{|l|l|l|l|l|}
\hline Characteristics & $\begin{array}{l}\text { Total } \\
\text { Number } \\
(\%)\end{array}$ & \multicolumn{2}{|l|}{$\begin{array}{l}\text { Non-SLN Metastasis } \\
(\%)\end{array}$} & \multirow{2}{*}{} \\
\cline { 2 - 4 } & No & Yes & \\
\hline $\begin{array}{l}\text { SLNM number, median } \\
\text { (range) }\end{array}$ & $0(0-5)$ & $0(0-0)$ & $1(0-5)$ & $<0.001$ \\
\hline
\end{tabular}

Abbreviations: IDC, invasive ductal carcinoma; HR, hormone receptor; NAC, neoadjuvant chemotherapy; AUS, axillary ultrasound; CR, complete response; PR, partial response; SD, stable disease; PD, progression disease; SLN, sentinel lymph node; SLNM, sentinel lymph node metastasis.

Thus, 13 of the 78 patients with residual nodal disease had a false-negative SLN finding (FNR: 16.7\%).

\section{Univariate Analysis of Factors Predicting the Risk of Non-SLN Metastasis}

Fifteen factors that could be determined prior to surgery were included in the univariate analysis (Table 1). The results showed that the frequency of non-SLN metastasis was markedly higher in patients with positive lymph nodes than in those with negative findings in post-NAC AUS (66.7\% vs $16.4 \%, p<0.001)$. In addition, non-SLN metastasis was more likely to be found in patients with a positive node than in those with a negative node prior to NAC $(28.6 \%$ vs $10.5 \%, p=0.001)$, and in patients with positive ER and PR than in those with negative ER and PR ( $29.8 \%$ vs $12.3 \%, p=0.001$ for ER; $29.1 \%$ vs $16.2 \%$, $p=0.021$ for PR). Low expression of Ki-67 was linked to more non-SLN metastasis ( $38.1 \%$ vs $19.3 \%, p=0.044)$. However, the risk of non-SLN metastasis did not exhibit a significant relationship with the expression of HER2 $(p=0.644)$. Therefore, the common molecular subtype which takes into account the expression of ER and PR, as well as HER2 and Ki-67 was not included in the analysis. Instead, the factor HR, which classified patients according to the expression of ER and PR, was included. Expectedly, patients with positive HR were at a higher risk of having non-SLN metastasis than those with negative HR $(28.3 \%$ vs $13.0 \%, p=0.004)$. Fewer SLNs and more SLNMs were associated with an increased likelihood of detecting residual disease in non-SLNs $(p<0.001$ for SLN number, $p<0.001$ for SLNM number). However, the risk of non-SLN metastasis was not correlated with age ( $p=0.723)$, menopause status $(p=0.402)$, pathological type $(p=0.311)$, clinical T stage $(p=0.130)$, and clinical tumor response $(p=0.806)$. 


\section{Multivariate Analysis of Factors Predicting the Risk of Non-SLN Metastasis}

Five factors with $p<0.05$ in the univariate analysis, namely nodal status prior to NAC, status of post-NAC AUS, HR expression, SLN number, and SLNM number were included in the multivariate analysis with the logistic regression backward stepwise method. The results showed that nodal status prior to NAC (odds ratio [OR]: 2.867, 95\% confidence interval $[\mathrm{CI}]: 1.198-6.862, p=0.018)$, post-NAC AUS status (OR: 4.933, 95\% CI: 1.605-15.128, $p=0.005$ ), SLN number (OR: 0.514, 95\% CI: 0.365-0.723, $p<0.001$ ), and SLNM number (OR: 2.800, 95\% CI: 1.$751-4.478, p<0.001)$ were independent predictors of nonSLN metastases (Table 2). Of note, HR expression $(p=0.516)$ was not identified as an independent predictor of non-SLN metastases.

\section{Predictive Model and Its Validation}

A model predicting the risk of non-SLN metastasis in patients with breast cancer receiving NAC was developed using variables with $p$-values $<0.05$ in the multivariate analysis as follows, with $P$ denoting the probability of non-SLN metastases:

$$
\begin{aligned}
\operatorname{Logit}(P)= & -0.885+1.053 a+1.596 b-0.666 c \\
& +1.030 d
\end{aligned}
$$

in which $a$ indicated nodal status prior to NAC, $b$ indicated the results of post-NAC AUS, $c$ indicated the SLN number, and $d$ indicated the SLNM number. The AUC for the model was 0.838 (95\% CI: $0.774-0.902, p<0.001)$, suggesting excellent discrimination (Figure 1).

The baseline characteristics of patients $(n=70)$ included in the testing set were comparable with those of patients assigned to the training set (Supplementary Table S1). ALND was performed in 40 patients (57.1\%, 40/70), including $18 \mathrm{SLN}+$ and $22 \mathrm{SLN}-$ patients. Non-SLN metastasis was found in nine patients; three of these were confined to the non-SLNs. The above predictive

Table 2 Multiple Analysis of Factors Predicting the Risk of NonSLN Metastasis

\begin{tabular}{|l|l|l|l|l|l|}
\hline Factors & B & SE & Wald & OR (95\% CI) & P \\
\hline Pre-NAC cN & 1.053 & 0.445 & 5.599 & $2.867(1.198-6.862)$ & 0.018 \\
Post-NAC AUS & 1.596 & 0.572 & 7.794 & $4.933(1.609-15.128)$ & 0.005 \\
SLN number & -0.666 & 0.175 & 14.552 & $0.514(0.365-0.723)$ & $<0.001$ \\
SLNM number & 1.030 & 0.240 & 18.478 & $2.800(1.751-4.478)$ & $<0.001$ \\
\hline
\end{tabular}

Abbreviations: NAC, neoadjuvant chemotherapy; AUS, axillary ultrasound; SLN sentinel lymph node; SLNM, sentinel lymph node metastasis.

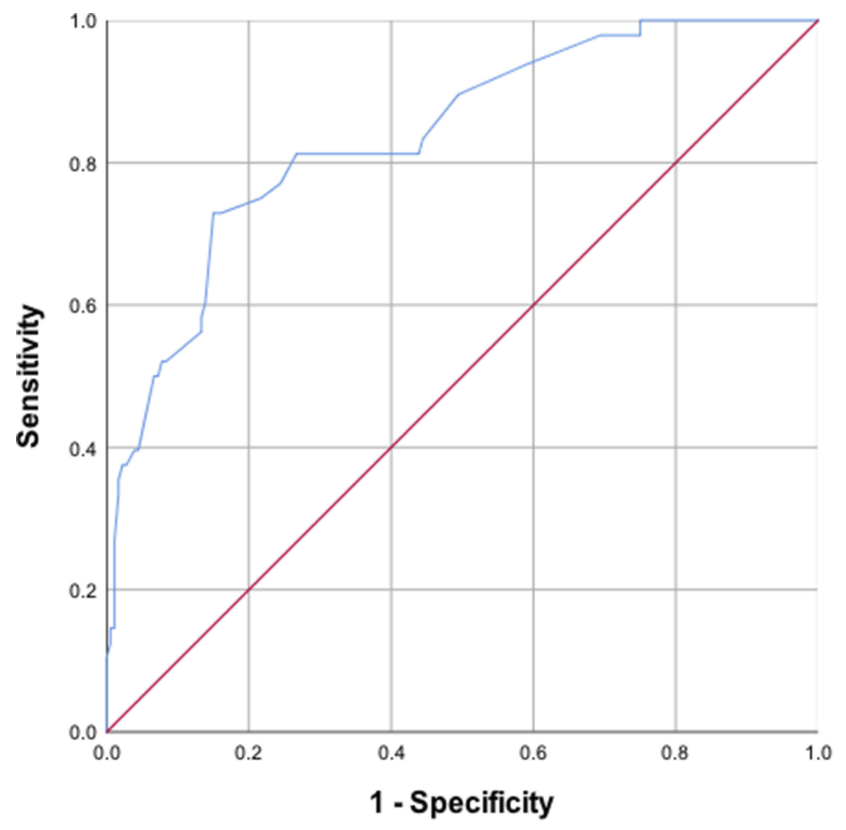

Figure I Receiver operating curve for the training set. The area under the curve was 0.838 ( $95 \% \mathrm{Cl}: 0.774-0.902, p<0.001)$.

model was applied to the testing set for internal validation, yielding an AUC of 0.808 (95\% CI: 0.609-1.000, $p=0.003$, Figure 2), thus demonstrating excellent discriminatory power. With a $30 \%$ cutoff value for predicted probability, this model had a sensitivity of $77.8 \%$, specificity of $96.4 \%$, accuracy of $87.1 \%$, negative predictive value of $96.4 \%$, positive predictive value of $50.0 \%$, and FNR of $12.5 \%$ (2/16).

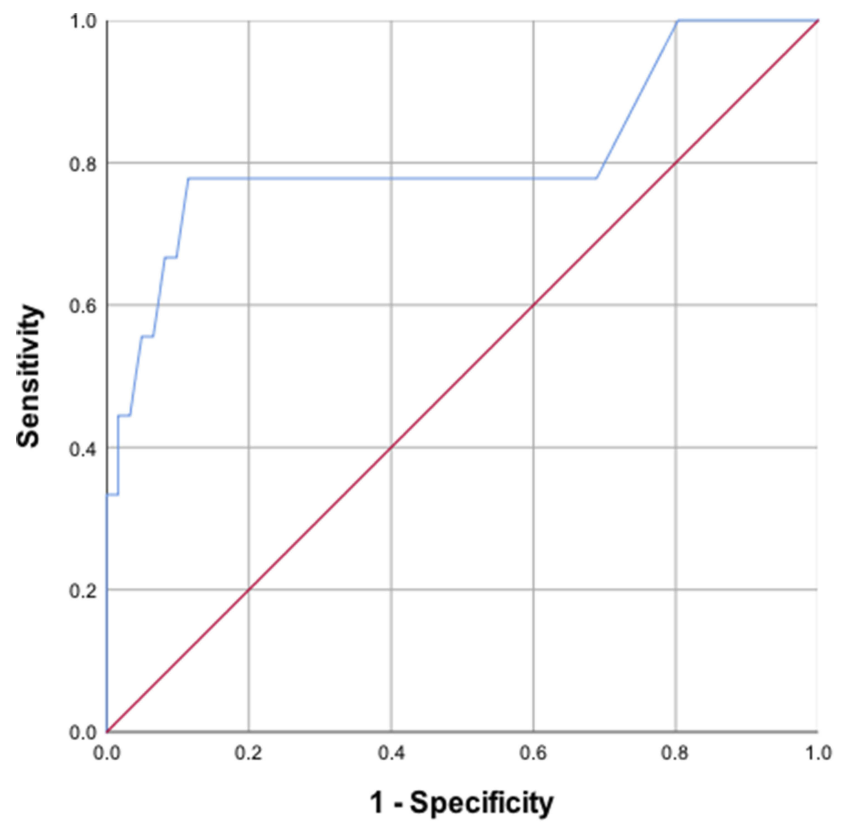

Figure 2 Receiver operating curve for the testing set. The area under the curve was 0.808 ( $95 \% \mathrm{Cl}: 0.609-1.000, p=0.003)$. 


\section{Discussion}

Initially, there was concern regarding the unacceptably high FNR for SLNB after NAC on account of fibrosis in lymphatic channels changing lymphatic drainage in the breast and nonuniform response to treatment in the axillary nodes. Based on this concern, ALND was previously the standard treatment for patients with breast cancer after NAC. However, ALND is associated with considerable morbidity that seriously affects the life quality of patients. Several prospective multicenter clinical trials were performed to evaluate the effectiveness of SLNB in patients with breast cancer receiving NAC. In recent years, SLNB has been increasingly used in the treatment of patients with breast cancer after NAC, aiming to minimize the extent of axillary surgery. Several methods have been developed to decrease the FNRs for SLNB after NAC, such as removing pre-NAC clipped node during $\mathrm{SLNB},{ }^{22,23}$ or performing SLNB only in patients with normal axilla detected through post-NAC AUS. ${ }^{24}$ While these researches had placed great value on the FNRs, a large majority of patients with positive SLNs, but without metastasis in non-SLNs, had to undergo unnecessary ALND. Furthermore, the results of the Z0011 25 and IBCSG $23-01^{26}$ trials indicated that patients with limited positive SLNs appear to have no survival benefit from ALND. Therefore, a predictive model such as the one developed in the present study to discriminate patients with or without metastasis in non-SLNs is of clinical significance.

Although previous studies suggested that pathological $\mathrm{T}$ stage, lymphovascular invasion, or extracapsular extension were significantly associated with the metastasis of nonSLNs, ${ }^{17,18}$ we excluded these from the analysis of this study as they could not be determined prior to breast surgery. In our medical center, all patients with breast cancer undergo preoperative AUS owing to its convenience and specificity. Several previous studies showed that a positive result on AUS indicated more extensive axillary nodal metastasis. ${ }^{27-30} \mathrm{Kim}$ et al reported that preoperative AUS could predict the risk of nonSLN metastasis and facilitate the selection of patients with early breast cancer in whom ALND could be omitted. ${ }^{31}$ It was also reported that the US could identify residual nodal disease after NAC with clearly defined standards. ${ }^{20}$ Thus, the US has been considered the most convenient and economic noninvasive method to evaluate the axilla, though it was not superior to other methods for the assessment of primary tumor response to NAC. ${ }^{32}$ Therefore, we added the post-NAC AUS status in the analysis of our study and demonstrated that it was the most significant predictor of non-SLN metastasis (OR: 4.933, $p=0.005$ ).
In addition to the post-NAC AUS status, the results of the multivariate analysis performed in this study showed that three other factors (i.e., nodal status prior to NAC, SLN number, and SLNM number) were the independent factors predicting the risk of non-SLN metastasis in patients with breast cancer after NAC. Indeed, both a positive axillary lymph node at presentation and greater SLNM number indicated heavier axillary burden. And the more SLNs were removed, the less likely metastasis would be identified in the rest of lymph nodes. Similarly, numerous previous studies have demonstrated the significant association between SLNM number and non-SLN metastasis. ${ }^{17,18,33}$ Furthermore, some clinical trials have reported a significant decrease in the FNRs with an increasing number of resected SLNs. ${ }^{5,14}$ Thus, nodal status prior to NAC and SLNM number were identified as the risk factors of nonSLN metastasis (OR: $2.867, p=0.018$ for nodal status prior to NAC, OR: $2.800, p<0.001$ for SLNM number), while SLN number was identified as the protective factor of non-SLN metastasis (OR: $0.514, p<0.001$ ).

Several models have been introduced in previous studies. ${ }^{16-18}$ However, these models included factors that could only be determined after breast surgery, such as pathological $\mathrm{T}$ stage, lymphovascular invasion, and extracapsular extension. Thus, they could not be used to facilitate the selection of patients who could avoid unnecessary ALND. In addition, these models had relatively limited discriminatory power with AUCs $<0.8$. Therefore, based on the results of the multivariate analysis conducted in this study, we developed a simple predictive model by including four significant factors that could be determined prior to breast surgery and yielded an AUC of 0.838 in the training set. The best cutoff of predicted probability according to the maximal Youden's index criteria was $30 \%$ for this model to discriminate patients who could avoid unnecessary ALND. This model was subsequently validated in an internal testing set and an AUC of 0.808 was achieved, demonstrating the reliable discriminatory power of this model.

There were several limitations to our study. Firstly, the SLNB method is correlated with the detection of SLNs. ${ }^{34}$ In our study, SLN mapping in all patients was conducted using only vital blue, which performs poorly in the identification of SLNs compared with radiolabeled colloid alone or blue dye. ${ }^{34}$ However, SLNB was successfully conducted in all patients in this study with at least one SLN removed. At least three SLNs were removed in $69.3 \%$ and $68.6 \%$ of patients in the training set and testing set, respectively, suggesting that the SLNB method was reliable in our center. Secondly, we 
validated this model internally. It requires further validation in patients from other centers before it can be widely used in the clinical setting. Thirdly, a proportion of the patients did not undergo ALND to obtain definitive pathological results of non-SLN. For these patients, we have to rely on follow-up imaging examination and US-guided FNA to evaluate the status of non-SLN. Finally, this was a single-center retrospective study, which inevitably leads to bias in the selection of patients. Additional multicenter prospective studies are warranted to verify the present results.

\section{Conclusion}

A new and simple model was developed to predict non-SLN metastasis in invasive breast cancer after NAC. It incorporated post-NAC AUS with other factors that could be determined prior to breast surgery. Thus, it was able to facilitate the selection of patients in whom unnecessary ALND could be omitted. This model performed excellently in the internal validation with an AUC of 0.808. However, it requires external validation in patients from other centers before it can be widely utilized in the clinical setting.

\section{Ethics Approval and Informed Consent}

This study was approved by the Ethics Committee of Fudan University Shanghai Cancer Center. Informed consent was waived due to its retrospective design and no identifiable information was disclosed.

\section{Abbreviations}

ALND, axillary lymph node dissection; AUC, area under the receiver operating characteristic curve; AUS, axillary ultrasound; $\mathrm{CI}$, confidence interval; $\mathrm{cN}+$, clinical positive axillary lymph node; ER, estrogen receptor; FNA, fine needle aspiration; FNR, false negative rate; HER2, human epidermal growth factor receptor 2; HR, hormone receptor; IDC, invasive ductal carcinoma; NAC, neoadjuvant chemotherapy; non-SLN, nonsentinel lymph nodes; OR, odds ratio; PR, progesterone receptor; SLN, sentinel lymph node; SLNB, sentinel lymph node biopsy; SLNM, sentinel lymph node metastasis; US, ultrasonography.

\section{Data Sharing Statement}

Please contact the corresponding author for data requests.

\section{Author Contributions}

Cai Chang and Kai Zhang conceived and designed the study. Kai Zhang and Qian Zhu wrote the manuscript. All authors contributed towards data analysis, drafting and critically revising the paper, gave final approval of the version to be published, and agreed to be accountable for all aspects of the work.

\section{Funding}

This study was supported by the National Natural Science Foundation of China (Grant Nos. 81627804 and 81830058).

\section{Disclosure}

The authors report no conflicts of interest in this work.

\section{References}

1. Recht A, Houlihan MJ. Axillary lymph nodes and breast cancer: a review. Cancer. 1995;76(9):1491-1512.

2. Ecanow JS, Abe H, Newstead GM, Ecanow DB, Jeske JM. Axillary staging of breast cancer: what the radiologist should know. Radiographics. 2013;33(6):1589-1612. doi:10.1148/rg.336125060

3. Krag DN, Anderson SJ, Julian TB, et al. Sentinel-lymph-node resection compared with conventional axillary-lymph-node dissection in clinically node-negative patients with breast cancer: overall survival findings from the NSABP B-32 randomised Phase 3 trial. Lancet Oncol. 2010;11(10):927-933.

4. Krag DN, Anderson SJ, Julian TB, et al. Technical outcomes of sentinel-lymph-node resection and conventional axillary-lymphnode dissection in patients with clinically node-negative breast cancer: results from the NSABP B-32 randomised Phase III trial. Lancet Oncol. 2007;8(10):881-888.

5. Kuehn T, Bauerfeind I, Fehm T, et al. Sentinel-lymph-node biopsy in patients with breast cancer before and after neoadjuvant chemotherapy (SENTINA): a prospective, multicentre cohort study. Lancet Oncol. 2013;14(7):609-618.

6. Boileau JF, Poirier B, Basik M, et al. Sentinel node biopsy after neoadjuvant chemotherapy in biopsy-proven node-positive breast cancer: the SN FNAC study. J Clin Oncol. 2015;33(3):258-264.

7. Boughey JC, Suman VJ, Mittendorf EA, et al. Sentinel lymph node surgery after neoadjuvant chemotherapy in patients with node-positive breast cancer: the ACOSOG Z1071 (Alliance) clinical trial. JAMA. 2013;310(14):1455-1461.

8. Caudle AS, Bedrosian I, Milton DR, et al. Use of sentinel lymph node dissection after neoadjuvant chemotherapy in patients with node-positive breast cancer at diagnosis: practice patterns of American Society of Breast Surgeons members. Ann Surg Oncol. 2017;24(10):2925-2934.

9. Srour MK, Tseng J, Luu M, Alban RF, Giuliano AE, Chung A. Patterns in the use of axillary operations for patients with node-positive breast cancer after neoadjuvant chemotherapy: a national cancer database (NCDB) analysis. Ann Surg Oncol. 2019;26(10):3305-3311.

10. Vriens B, Keymeulen K, Kroep JR, et al. Axillary staging in breast cancer patients treated with neoadjuvant chemotherapy in two Dutch phase III studies. Oncotarget. 2017;8(28):46557-46564. doi:10.18632/ oncotarget.v8i28

11. Alvarado R, Yi M, Le-Petross H, et al. The role for sentinel lymph node dissection after neoadjuvant chemotherapy in patients who present with node-positive breast cancer. Ann Surg Oncol. 2012;19 (10):3177-3184. doi:10.1245/s10434-012-2484-2 
12. Classe JM, Bordes V, Campion L, et al. Sentinel lymph node biopsy after neoadjuvant chemotherapy for advanced breast cancer: results of Ganglion Sentinelle et Chimiotherapie Neoadjuvante, a French prospective multicentric study. J Clin Oncol. 2009;27(5):726-732. doi:10.1200/JCO.2008.18.3228

13. Newman EA, Sabel MS, Nees AV, et al. Sentinel lymph node biopsy performed after neoadjuvant chemotherapy is accurate in patients with documented node-positive breast cancer at presentation. Ann Surg Oncol. 2007;14(10):2946-2952. doi:10.1245/s10434-007-9403-y

14. Mamounas EP, Brown A, Anderson S, et al. Sentinel node biopsy after neoadjuvant chemotherapy in breast cancer: results from National Surgical Adjuvant Breast and Bowel Project Protocol B-27. J Clin Oncol. 2005;23(12):2694-2702. doi:10.1200/JCO.2005.05.188

15. Reitsamer R, Peintinger F, Rettenbacher L, Prokop E. Sentinel lymph node biopsy in breast cancer patients after neoadjuvant chemotherapy. J Surg Oncol. 2003;84(2):63-67. doi:10.1002/(ISSN)1096-9098

16. Jeruss JS, Newman LA, Ayers GD, et al. Factors predicting additional disease in the axilla in patients with positive sentinel lymph nodes after neoadjuvant chemotherapy. Cancer. 2008;112 (12):2646-2654. doi:10.1002/(ISSN)1097-0142

17. Ryu JM, Lee SK, Kim JY, et al. Predictive factors for nonsentinel lymph node metastasis in patients with positive sentinel lymph nodes after neoadjuvant chemotherapy: nomogram for predicting nonsentinel lymph node metastasis. Clin Breast Cancer. 2017;17(7):550-558. doi:10.1016/j.clbc.2017.03.014

18. Yang Y, He Y, Fan Z, Liu Y, Ouyang T. A new model to predict risk of nonsentinel lymph node status in Chinese sentinel lymph node-positive patients after neoadjuvant chemotherapy. Breast J. 2018;24(6):883-888. doi:10.1111/tbj.2018.24.issue-6

19. Peppe A, Wilson R, Pope R, Downey K, Rusby J. The use of ultrasound in the clinical re-staging of the axilla after neoadjuvant chemotherapy (NACT). Breast. 2017;35:104-108. doi:10.1016/j. breast.2017.05.015

20. Le-Petross HT, McCall LM, Hunt KK, et al. Axillary ultrasound identifies residual nodal disease after chemotherapy: results from the American College of Surgeons Oncology Group Z1071 trial (Alliance). AJR Am J Roentgenol. 2018;210(3):669-676. doi:10.2214/AJR.17.18295

21. Ibrahim-Zada I, Grant CS, Glazebrook KN, Boughey JC. Preoperative axillary ultrasound in breast cancer: safely avoiding frozen section of sentinel lymph nodes in breast-conserving surgery. $J$ Am Coll Surg. 2013;217(1):7-15; discussion 15-16. doi:10.1016/j. jamcollsurg.2013.01.064

22. Boughey JC, Ballman KV, Le-Petross HT, et al. Identification and resection of clipped node decreases the false-negative rate of sentinel lymph node surgery in patients presenting with node-positive breast cancer (T0-T4, N1-N2) who receive neoadjuvant chemotherapy: results from ACOSOG Z1071 (Alliance). Ann Surg. 2016;263 (4):802-807. doi:10.1097/SLA.0000000000001375
23. Diego EJ, McAuliffe PF, Soran A, et al. Axillary staging after neoadjuvant chemotherapy for breast cancer: a pilot study combining sentinel lymph node biopsy with radioactive seed localization of pre-treatment positive axillary lymph nodes. Ann Surg Oncol. 2016;23(5):1549-1553. doi:10.1245/s10434-015-5052-8

24. Boughey JC, Ballman KV, Hunt KK, et al. Axillary ultrasound after neoadjuvant chemotherapy and its impact on sentinel lymph node surgery: results from the American College of Surgeons Oncology Group Z1071 trial (Alliance). J Clin Oncol. 2015;33(30):3386-3393. doi:10.1200/JCO.2014.57.8401

25. Giuliano AE, Ballman KV, McCall L, et al. Effect of axillary dissection vs no axillary dissection on 10-year overall survival among women with invasive breast cancer and sentinel node metastasis: the ACOSOG Z0011 (Alliance) randomized clinical trial. JAMA. 2017;318(10):918-926. doi:10.1001/jama.2017.11470

26. Galimberti V, Cole BF, Viale G, et al. Axillary dissection versus no axillary dissection in patients with breast cancer and sentinel-node micrometastases (IBCSG 23-01): 10-year follow-up of a randomised, controlled phase 3 trial. Lancet Oncol. 2018;19(10):1385-1393.

27. Farrell TP, Adams NC, Stenson M, et al. The Z0011 trial: is this the end of axillary ultrasound in the pre-operative assessment of breast cancer patients? Eur Radiol. 2015;25(9):2682-2687.

28. Moorman AM, Bourez RL, Heijmans HJ, Kouwenhoven EA. Axillary ultrasonography in breast cancer patients helps in identifying patients preoperatively with limited disease of the axilla. Ann Surg Oncol. 2014;21(9):2904-2910.

29. Nath J, Sami N, Massey J, Donnelly J, Corder AP. Selection for axillary clearance in breast cancer (ultrasound negative, sentinel node positive patients have low rates of further metastases). Eur J Surg Oncol. 2013;39(5):450-454.

30. Reyna C, Kiluk JV, Frelick A, Khakpour N, Laronga C, Lee MC. Impact of axillary ultrasound (AUS) on axillary dissection in breast conserving surgery (BCS). J Surg Oncol. 2015;111(7):813-818.

31. Kim GR, Choi JS, Han BK, et al. Preoperative axillary US in early-stage breast cancer: potential to prevent unnecessary axillary lymph node dissection. Radiology. 2018;288(1):55-63.

32. Vriens BE, de Vries B, Lobbes MB, et al. Ultrasound is at least as good as magnetic resonance imaging in predicting tumour size post-neoadjuvant chemotherapy in breast cancer. Eur $J$ Cancer. 2016;52:67-76.

33. Maimaitiaili A, Wu D, Liu Z, Liu H, Muyiduli X, Fan Z. Analysis of factors related to non-sentinel lymph node metastasis in 296 sentinel lymph node-positive Chinese breast cancer patients. Cancer Biol Med. 2018;15(3):282-289.

34. Boughey JC, Suman VJ, Mittendorf EA, et al. Factors affecting sentinel lymph node identification rate after neoadjuvant chemotherapy for breast cancer patients enrolled in ACOSOG Z1071 (Alliance). Ann Surg. 2015;261(3):547-552.

\section{Publish your work in this journal}

Cancer Management and Research is an international, peer-reviewed open access journal focusing on cancer research and the optimal use of preventative and integrated treatment interventions to achieve improved outcomes, enhanced survival and quality of life for the cancer patient.
The manuscript management system is completely online and includes a very quick and fair peer-review system, which is all easy to use. Visit http://www.dovepress.com/testimonials.php to read real quotes from published authors. 